\title{
Genetic analysis in a compound heterozygote family with familial hypercholesterolemia
}

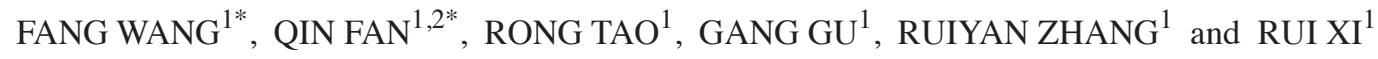 \\ ${ }^{1}$ Department of Cardiology, Rui Jin Hospital; ${ }^{2}$ Institute of Cardiovascular Diseases, \\ Shanghai Jiao Tong University School of Medicine, Shanghai 200025, P.R. China
}

Received September 8, 2017; Accepted February 19, 2018

DOI: $10.3892 / \mathrm{mmr} .2018 .8904$

\begin{abstract}
Homozygous familial hypercholesterolemia (FH) is rare, with an incidence of $\sim$ one in a million and commonly presents with a genetic mutation. The genetic variations of families with FH were clinically analyzed to investigate the association between the phenotype and genotype of patients. Direct sequencing was conducted for the proband and her parents to detect mutations in the fragment of 18 exons of the low-density lipoprotein receptor (LDLR) and apolipoprotein B100 Q3500R in the peripheral blood genomic DNA. The gene sequences were compared with normal ones to find mutations using GenBank. The QX200 Droplet Digital PCR system was used to detect target DNA copy number variations of the proband and her parents. The functional alterations resulting from the novel mutations were verified by quantitative polymerase chain reaction, western blotting and flow cytometric analyses. The lipid levels of the proband and her parents were all elevated. Genetic testing results indicated that the proband and her mother had a novel heterozygous missense mutation $(\mathrm{C} 377 \mathrm{G}, 28893 \mathrm{~T}>\mathrm{G})$ in exon 8 of the LDLR gene, whereas the proband and her father had LDLR gene DNA fragment deletions in exon 18. Clinically, the proband was of a compound heterozygous genotype and her parents were of the simple heterozygous genotype. Furthermore, both mutations led to impaired expression and LDL binding and internalization function of LDLR in vitro. The proband's genotype was confirmed to be compound heterozygous $\mathrm{FH}$, leading to clinical manifestations in line with the homozygous FH phenotype. The phenotype is highly associated with the genotype in this type of compound heterozygous FH.
\end{abstract}

Correspondence to: Dr Rui Xi, Department of Cardiology, Rui Jin Hospital, Shanghai Jiao Tong University School of Medicine, 197 Rui Jin Road II, Shanghai 200025, P.R. China

E-mail: xixi76120@163.com

${ }^{*}$ Contributed equally

Key words: compound heterozygous familial hypercholesterolemia, low-density lipoprotein receptor gene, missense mutations, in-frame deletion

\section{Introduction}

Familial hypercholesterolemia [FH; Online Mendelian Inheritance in Man (OMIM) reference no. 143890] is one of the most common and most serious autosomal dominant, hereditary monogenic diseases. Clinically, it is divided into two types: Homozygous and heterozygous. Homozygous FH patients manifest rising plasma low-density lipoprotein cholesterol (LDL-C), tendon xanthoma in infancy or early childhood, early onset atherosclerosis, severe coronary heart disease, or even death during youth and its incidence is $\sim$ one in a million (1). Heterozygous FH patients' phenotypic features are not obvious and it has an incidence rate of $\sim 1$ in 500 (2). The most reliable diagnosis of FH may be made using phenotypic criteria and genetic testing. Genetic testing increases the accuracy of an FH diagnosis. The pathology of FH is associated with serum LDL-C metabolic pathway abnormalities. Generally, FH results from mutations in the genes encoding the LDL receptor (LDLR), apolipoprotein $B(a p o B)$, or proprotein convertase subtilisin/kexintype 9 (PCSK9). Previous studies have identified several LDLR mutations in FH and the association between the phenotype and underlying genotype $(3,4)$, including a systematic review analyzing the characteristics, distribution, gene frequency and association between the genotype and phenotype of LDLR mutations especially in the Chinese population (5). However, FH is a very heterogeneous disease and there are different variants of LDLR gene mutations throughout the gene. Although the phenotype is supposed to be associated with the genotype, $\sim 30 \%$ of patients with the typical clinical phenotype still cannot be identified with significant pathogenic genetic mutations $(4,6-8)$, which may be due to the extensive genetic heterogeneity of FH patients or its clinical variability. Therefore, it is challenging and essential to diagnose an FH patient with homozygous or heterozygous FH. In the present study, the genetic variations in a family with compound heterozygous FH were analyzed to investigate the association between the phenotype and the genotype.

\section{Materials and methods}

Subjects. All the investigated subjects gave written informed consent for all tests and the publication of associated data and accompanying images, prior to taking part in the study and participating in these examinations. The study was conducted 
according to the Helsinki declaration (2013) and approved by the Institutional Ethics Committees of Shanghai Jiao Tong University, affiliated to Rui Jin Hospital (Shanghai, China).

Determination of lipids. Blood samples (20 $\mathrm{ml}$ per patient) were obtained from the proband and her parents following a $12 \mathrm{~h}$ fast at Rui Jin Hospital in March, 2014. The proband was 16 years old and her parents were both 47 years old. All samples were collected simultaneously, immediately placed on ice and subsequently centrifuged within $30 \mathrm{~min}$ at $1,000 \mathrm{x} \mathrm{g}$ at $4^{\circ} \mathrm{C}$ for $20 \mathrm{~min}$. Total cholesterol (TC), triglycerides (TG), LDL-C and high-density lipoprotein cholesterol (HDL-C) were measured using routine commercial kits (Beckman Coulter, Inc., Brea, CA, USA) and a Beckman AU 4500 automated biochemical analyzer (Beckman Coulter, Inc.), according to the manufacturer's protocols.

Genotyping assays. Venous peripheral blood was collected from the proband and her parents. Genomic DNA was prepared from the peripheral white blood cells by phenol/chloroform extraction (9). The polymerase chain reaction (PCR) and direct sequencing were used to detect the fragment of 18 exons of the low-density lipoprotein receptor (LDLR; OMIM 606945) and apolipoprotein B100 (apoB100) Q3500R of the proband and her parents. The primer pairs for these gene fragments are presented in Table I. The gene sequences were compared with normal sequences downloaded from GenBank (https://www. ncbi.nlm.nih.gov/nuccore/DQ379956.1) to identify mutations.

Detection of target DNA copy number variations. The QX200 Droplet Digital PCR $\left(\right.$ ddPCR $\left.^{\mathrm{TM}}\right)(10,11)$ system was used to detect target DNA copy number variations of the proband and herparents. The workflow was carried out based on the manufacturer's protocol (http://www.bio-rad.com/zh-cn/product/qx20 0 -droplet-digital-pcr-system). The TaqMan ${ }^{\circledR}$ Copy Number Reference Assay, with RNase P (Applied Biosystems; Thermo Fisher Scientific, Inc., Waltham, MA, USA), is recommended as the standard reference assay for copy number analysis, which is present at 1 copy per haploid genome and was labeled with VIC fluorescent dye. The 1st target site was located at exon 18 of the LDLR gene. The forward primer was 5'-TTG GCAGAGACAGATGGTCAGT-3' and the reverse primer was 5'-CGGGACTCCAGGCAGATG-3'; the probe was FAMTGG AGGATGACGTGGCGTMGBNFQ. The 2nd target site was located at a distal region of the exon 18. The forward primer was 5'-GATTGACGAAATTGGTGCTCAA-3' and the reverse primer was 5'-GCCATCTGAGCCCGCTTA-3'; the probe was FAMCAAGTGGCAAACAGGMGBNFQ.

CopyCaller $^{\mathrm{TM}}$ Software, v2.0 (Thermo Fisher Scientific, Inc.), was used to analyze the ratios of the FAM and VIC fluorescence number, including RNase P in the 1st target site and that in the 2nd target site, respectively. The ratios obtained represented the DNA copy number at the target site, compared with a haploid genome.

Cell culture, plasmid construction and transfection. The LDLR wild-type gene was cloned as well as genes with specific mutations including C377G and L855fs, from the GV230 vector (Shanghai GeneChem, Co., Ltd., Shanghai, China) separately, which has an $\mathrm{N}$-terminal green fluorescent protein
(GFP) tag. Prior to being used, the integrity of all constructs were verified by quantitative (q)PCR and western blot analysis.

The wild type (WT) and mutant plasmids $(20 \mu \mathrm{g} / \mathrm{ml})$ were transfected into 293T cells $\left(4 \times 10^{5} / \mathrm{ml}\right.$; Shanghai GeneChem, Co., Ltd.), which do not have endogenous LDL-R expression, using Lipofectamine 2000 (Thermo Fisher Scientific, Inc.), according to the manufacturer's protocol. One day prior to transfection, cells $\left(2 \times 10^{5}\right.$ cells/well) were seeded in a six-well plates. Transfection efficiency was estimated by counting the GFP positive cells using a fluorescent microscope, which was observed to be $\sim 65-70 \%$ in all experiments, following the counting of 7 randomly selected fields of view (magnification, $\mathrm{x} 400$ ). Transfected cells were cultured in RPMI 1640 (cat. no. 22400-089; Gibco; Thermo Fisher Scientific, Inc.) supplemented with $10 \%$ lipoprotein deprived serum (Gibco; Thermo Fisher Scientific, Inc.) for $48 \mathrm{~h}$ prior to analysis of LDLR expression and function by flow cytometry. Cells were maintained at $37^{\circ} \mathrm{C}$ and a humidity of $95 \%$ in an incubator with an atmosphere of $5 \% \mathrm{CO}_{2}$.

Reverse transcription ( $R T)$ - $q P C R$ analysis of $L D L R$. Total RNA was isolated from the cultured 293T cells using the acid-phenol extraction method in the presence of chaotropic salts (TRIzol ${ }^{\mathrm{TM}}$; Thermo Fisher Scientific, Inc.) and subsequent isopropanol-ethanol precipitation. Reverse transcription of mRNA encoding LDLR as well as GAPDH was performed on $2 \mu \mathrm{g}$ total RNA using the SuperScript First-Strand Synthesis System (Thermo Fisher Scientific, Inc.), following the manufacturer's protocol. qPCR was conducted using the SYBR Green PCR Master Mix (Applied Biosystems; Thermo Fisher Scientific, Inc.) and ABI Prism 7500 Sequence Detection System (Applied Biosystems; Thermo Fisher Scientific, Inc.), according to the manufacturer's protocol. The thermocycling conditions included initial activation at $95^{\circ} \mathrm{C}$ for $10 \mathrm{~min}$, followed by 40 cycles of denaturation for $15 \mathrm{sec}$ at $95^{\circ} \mathrm{C}$ and an annealing/extension step for $1 \mathrm{~min}$ at $60^{\circ} \mathrm{C}$. The equation $2^{-\Delta \Delta \mathrm{Cq}}$ measurement was used to analyze the results (12). Primer sets were as follows: LDL-R: Forward, 5'-AACGAATGCTTG GACAACAAC-3', reverse 5'-CTTCCTCACACTGGCACT TG-3'. GAPDH: Forward, 5'-TGACTTCAACAGCGACAC CCA-3', reverse 5'-CACCCTGTTGCTGTAGCCAAA-3'.

Western blot analysis. Cells were lysed in a radioimmunoprecipitation assay lysis buffer containing $100 \mathrm{mM} \mathrm{NaCl}$ and 10 nM EDTA (cat. no. 9803; Cell Signaling Technology, Inc., Danvers, MA, USA). Protein concentration was determined using the BCA assay and following this, protein samples $(15 \mu \mathrm{g})$ were loaded and separated by $10 \%$ SDS-PAGE and transferred onto nitrocellulose membranes. Non-specific binding sites were blocked in Tris-buffered saline/Tween buffer (TBST; $0.1 \%, \mathrm{v} / \mathrm{v}$ ) supplemented with 5\% non-fat dry milk for $1 \mathrm{~h}$ at room temperature and the membrane was then probed with primary antibodies to LDLR (1:5,000; cat. no. ab52818; Abcam, Cambridge, UK) and GAPDH (1:2,000; cat. no. sc-32233; Santa Cruz Biotechnology, Inc., Dallas, TX, USA) in TBST supplemented with $1 \%$ bovine serum albumin (BSA; Beyotime Biotechnology, Shanghai, China) at $4^{\circ} \mathrm{C}$ overnight. Following three subsequent washes in TBST, membranes were incubated with the following horseradish peroxidase (HRP)-conjugated secondary antibodies for 
Table I. Primers of the gene fragments and amplification fragment length of each polymerase chain reaction system.

\begin{tabular}{|c|c|c|c|}
\hline Amplified segments & Upstream primer $\left(5^{\prime}-3^{\prime}\right) \mathrm{F}$ & Downstream primer $\left(5^{\prime}-3^{\prime}\right) \mathrm{R}$ & Fragment length (bp) \\
\hline ApoB-100E26 & gtggagggtagtcataacag & acatacaagcaaagggaccg & 510 \\
\hline LDLR promoter & gagtgggaatcagagcttcacgggt & cattgaaatgctgtaaatgacgtgg & 155 \\
\hline LDLR E1 & acccaaatacaacaaatcaagtcg & agggtgagggaccgcatct & 629 \\
\hline LDLR E2 & ttagttggcaggaaatagaca & gccaccacctctatcttgt & 598 \\
\hline LDLR E3 & gectcagtgggtctttcc & aatgacccacgtaacatgaag & 563 \\
\hline LDLR E4-1 & atagaatgggctggtgttgg & aggacaaatctgacgaggaa & 440 \\
\hline LDLR E4-2 & cgaagatggctcgatga & gcttattgggaaatcactgttt & 466 \\
\hline LDLR E5 & aaggtggcacgattatggc & cttctcagagcctcacttcctt & 566 \\
\hline LDLR E6 & gattacaggcacaaaccacc & ggaaagggactgagacatga & 339 \\
\hline LDLR E7 & attagcctgtcatggtcgtg & aacaccttgggacgccga & 703 \\
\hline LDLR E8 & gggtgcgtaatccctgtaa & ttaggacttgggcttgcc & 834 \\
\hline LDLR E9 & tttctgggtgcctcctctg & ggaatggagggagcagga & 655 \\
\hline LDLR E10 & tccatcgectacctcttctt & tggttagtgggctgggca & 564 \\
\hline LDLR E11 & aaaacccaaacaagccaca & ctgetccetgaaggtttcc & 381 \\
\hline LDLR E12 & aggtatgttcgcaggacagc & ccttctccttggecgtct & 784 \\
\hline LDLR E13 & ccagtgtttaacgggatttg & tgtgagaggaccaccctgag & 353 \\
\hline LDLR E14 & tcttccacaacctcacccag & ctgctcatctgtcaaatgggta & 399 \\
\hline LDLR E15 & tgagactttcgtcattaggcg & gtctaataacagttcttgecetc & 448 \\
\hline LDLR E16 & tgggaagttctccaagtgtc & gtcccgtgctgtggaatag & 435 \\
\hline LDLR E17 & caaggttatggtacgatgcc & cagaatgtcagcggacaatg & 418 \\
\hline LDLR E18 & actcaccgtctccetctgg & gggagccagaacaggctt & 333 \\
\hline
\end{tabular}

F, forward; R, reverse; LDLR, low-density lipoprotein; ApoB-100, apolipoprotein 100.

$80 \mathrm{~min}$ at room temperature: Anti-rabbit HRP (cat. no. 7074; 1:3,000; Cell Signaling Technology, Inc.) and anti-mouse HRP (cat. no. 7076; 1:3,000; Cell Signaling Technology, Inc.). Following another three washes in TBST, specific bands were detected using an enhanced chemiluminescent reagent (cat. no. B18005; Selleck Chemicals, Houston, TX, USA), and the obtained images were analyzed using ImageJ (version 1.48; National Institutes of Health, Bethesda, MD, USA).

Flow cytometric analysis. Following transfection, cells $\left(1 \times 10^{6}\right)$ were harvested by trypsinization and suspended by PBS for flow cytometric analysis. The cell suspension was then divided into two groups: The first group was used for LDL binding ability analysis; the second was used for LDL internalization analysis. The cells undergoing LDL binding analysis were placed in 20mg/ml1,1'-Dioctadecyl-3,3,3',3'-tetramethylindocarbocyanine perchlorate (Dil) labeled LDL (Molecular Probes; Thermo Fisher Scientific, Inc.) for $1 \mathrm{~h}$ at $4^{\circ} \mathrm{C}$. Following the incubation, the medium was removed, then the cells were washed twice in ice-cold PBS with 0.5\% BSA (Beyotime Biotechnology, Inc.). The same cells were incubated in $20 \mathrm{mg} / \mathrm{ml}$ Dil labeled LDL for $1 \mathrm{~h}$ at $37^{\circ} \mathrm{C}$ for LDLR internalization analysis, followed by medium removal. Following that, cells were washed twice in ice-cold PBS with $0.5 \%$ BSA prior to flow cytometry analysis. LDLR function was analyzed by a BD FACS Flow Cytometer with an argon laser operating at $514 \mathrm{~nm}$ (Dil). All experiments and analyses were repeated three times. For analyses, histogram plots presented Dil-LDL positive cells gated on GFP tagged LDL-R and the x-axis demonstrated the activity of Dil-LDL binding and of Dil-LDL internalization. All data were collected and analyzed using FlowJo (version 9.3.2; FlowJo LLC, Ashland, OR, USA).

Statistical analysis. Data are presented as mean \pm standard error of the mean. Comparisons between two groups were made using a Student's t-test or Mann-Whitney U test, while the data obtained from multiple groups were compared using Kruskal-Wallis test with Dunn's multiple comparison test. A minimum of three repeats per experiment was performed. All data were analyzed using SPSS software, version 20.0 (IBM SPSS, Armonk, NY, USA). $\mathrm{P}<0.05$ was considered to indicate a statistically significant difference.

\section{Results}

Clinical features of the proband and her family members. The proband was a 16-year-old girl, who presented with elevated serum lipid levels and a xanthoma for $\sim 13$ years. At the age of 3 , her parents identified a soy bean-sized xanthoma on the joints of her limbs and her lab results demonstrated an abnormally high level of serum lipids. The proband was prescribed Lipitor therapy, $10 \mathrm{mg}$ orally once daily for 4 years, with accompanying dietary and lifestyle alterations. However, her serum lipid levels remained high, with a TC of $20.15 \mathrm{mmol} / \mathrm{l}$ and LDL-C of $13.16 \mathrm{mmol} / \mathrm{l}$. Her xanthoma also increased gradually and therefore, she was prescribed more anti-hyperlipidemic drugs: Lipitor, $20 \mathrm{mg}$ twice a day, along with $4 \mathrm{~g}$ cholestyramine thrice a day. The lipid levels were still 
poorly controlled, although the growth rate of the xanthoma slowed slightly.

A physical examination revealed that the proband was of good nutritional status and normal development, and her BMI was $20.7 \mathrm{~kg} / \mathrm{m}^{2}$. Flat or nodular, yellow protruding rashes, $5-10 \mathrm{~cm}$ in diameter, were visible on her Achilles tendon, knees, elbows and ears (Fig. 1). Systolic murmurs of the left carotid artery were detected and a jet-like murmur around the aortic valve area was heard.

Echocardiography demonstrated severe, supravalvular aortic stenosis $7 \mathrm{~mm}$ from the aortic root. The narrowest diameter was $\sim 5 \mathrm{~mm}$ and there were multiple echogenic plaques on the irregular aortic arch sidewall. Vascular ultrasonography demonstrated bilateral carotid atherosclerotic plaque formation. The percentage stenosis of the right internal carotid artery was $\sim 50 \%$ and the percentage occlusion of the left internal carotid artery was $\sim 60 \%$. The right common carotid artery had a soft plaque of $\sim 2.6 \times 27.2 \mathrm{~mm}$ and left common carotid artery had a soft plaque of $\sim 2.8 \times 16.4 \mathrm{~mm}$. Coronary computed tomography angiography demonstrated that there were multiple plaques on the wall of the left main stem, the middle of the left anterior descending artery and the right coronary with mild luminal stenosis; a mixed plaque was also seen on the proximal left circumflex artery with moderate luminal stenosis. The aortic root had multiple plaques, leading to supravalvularaortic stenosis.

Furthermore, the proband's parents had no apparent clinical features upon physical examination. They had a non-consanguineous marriage and the family had no similar diseases. The proband had no secondary causes of hypercholesterolemia, including proteinuria, hypothyroidism or medications.

The proband's lipid values remained high, with a TC of $14.21 \mathrm{mmol} / \mathrm{l}$, a LDL-C of $12.22 \mathrm{mmol} / 1$ and a TG of $1.63 \mathrm{mmol} / \mathrm{l}$, even following treatment with $40 \mathrm{mg}$ atorvastatin per day. Although her parents had no apparent clinical features upon physical examination, they also suffered from hyperlipidemia and their serum lipid levels were mildly elevated, accompanied by high TC and LDL-C levels. The results of the serum lipid profile of the primary family members are presented in Table II.

Genetic mutations. The genetic screening results demonstrated that the proband and her mother had the same heterozygous missense mutation $\mathrm{C} 377 \mathrm{G}, 28893 \mathrm{~T}>\mathrm{G}$ in exon 8 (Fig. 2A and B), whereas her father did not have this mutation (Fig. 2C). Therefore, the proband does not have a homozygous mutation in her genotype. However, it was demonstrated that in exons 16 and 18 of the proband and her parents, gene sequences were mismatched. Multiple AA alleles were present in the father, whereas GG alleles were present in the mother and proband (rs13306501, rs2304182, rs2116899, rs2116897, rs2116898 and rs14158; Table III), which didn't obey the law of inheritance. According to the laws of inheritance, if the DNA fragment was complete in the associated exon region of the father, AG alleles would be expected to be present in the proband (A from her father and $\mathrm{G}$ from her mother). However, GG alleles were present in both the mother and proband, which suggests that the proband did not inherit said alleles in the same region from her father as
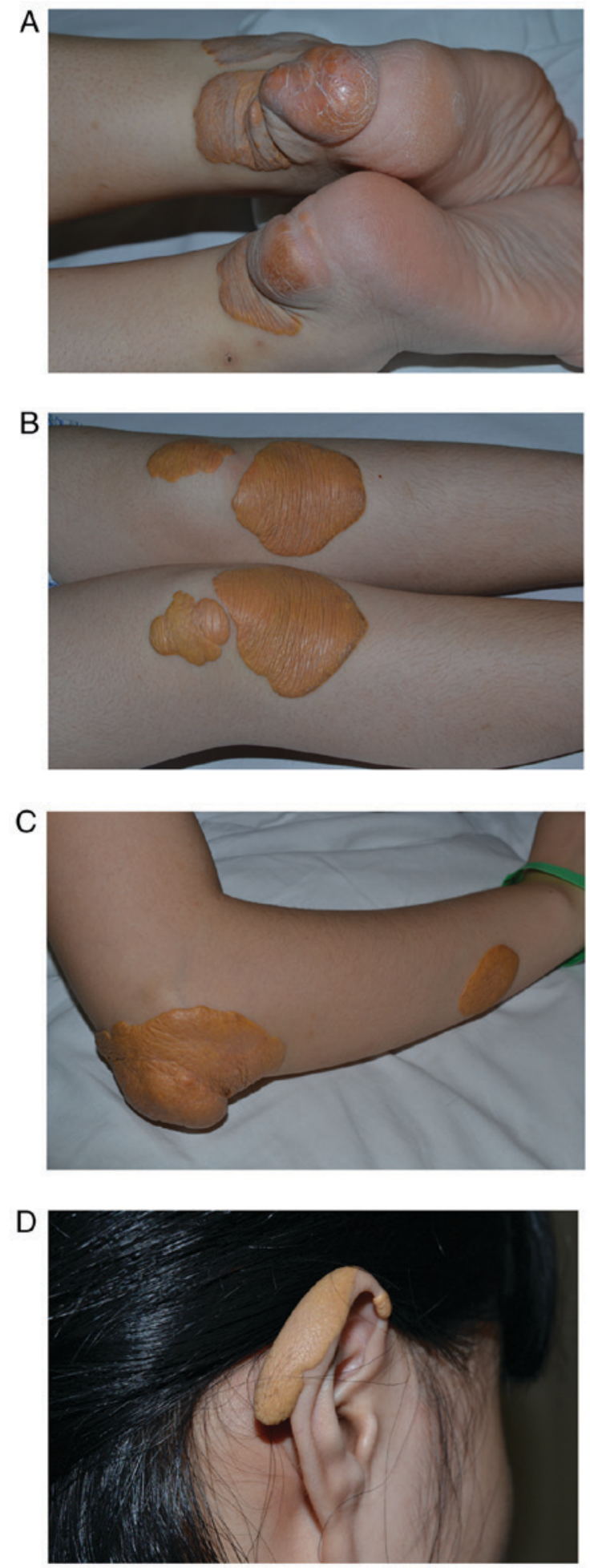

Figure 1. Clinical manifestations of the proband. Photos of the xanthomas in different locations of the proband. (A) Achilles tendon xanthomas. (B) Skin xanthomas on the knees. (C) Cutaneous xanthomas on the elbow region. (D) Skin xanthomas on the ear.

she did from her mother. As such, it was hypothesized that a large DNA fragment had been deleted in the exon 16 and 18 regions of both the father and the proband. There was no mutation observed in apoBQ3500R.

Abnormal target DNA copy number. The results of the ddPCR $^{\mathrm{TM}}$ system demonstrated that the ratios of the father 
Table II. Serum lipid profile of primary family members.

\begin{tabular}{lcccccc}
\hline Subjects & Age (year) & TC (mmol/l) & HDL-C (mmol/l) & LDL-C (mmol/l) & TG (mmol/l) & Treatment \\
\hline Proband & 16 & 14.21 & 0.84 & 12.22 & $\begin{array}{l}\text { Atorvastatin } \\
40 \text { mg/day }\end{array}$ \\
Father & 47 & 7.34 & 1.35 & 4.40 & 1.4 & None \\
Mother & 47 & 8.33 & 1.79 & 4.98 & 0.97 & None \\
\hline
\end{tabular}

TC, total cholesterol; HDL-C, high-density lipoprotein cholesterol; TG, triglycerides; LDL-C, low-density lipoprotein cholesterol.

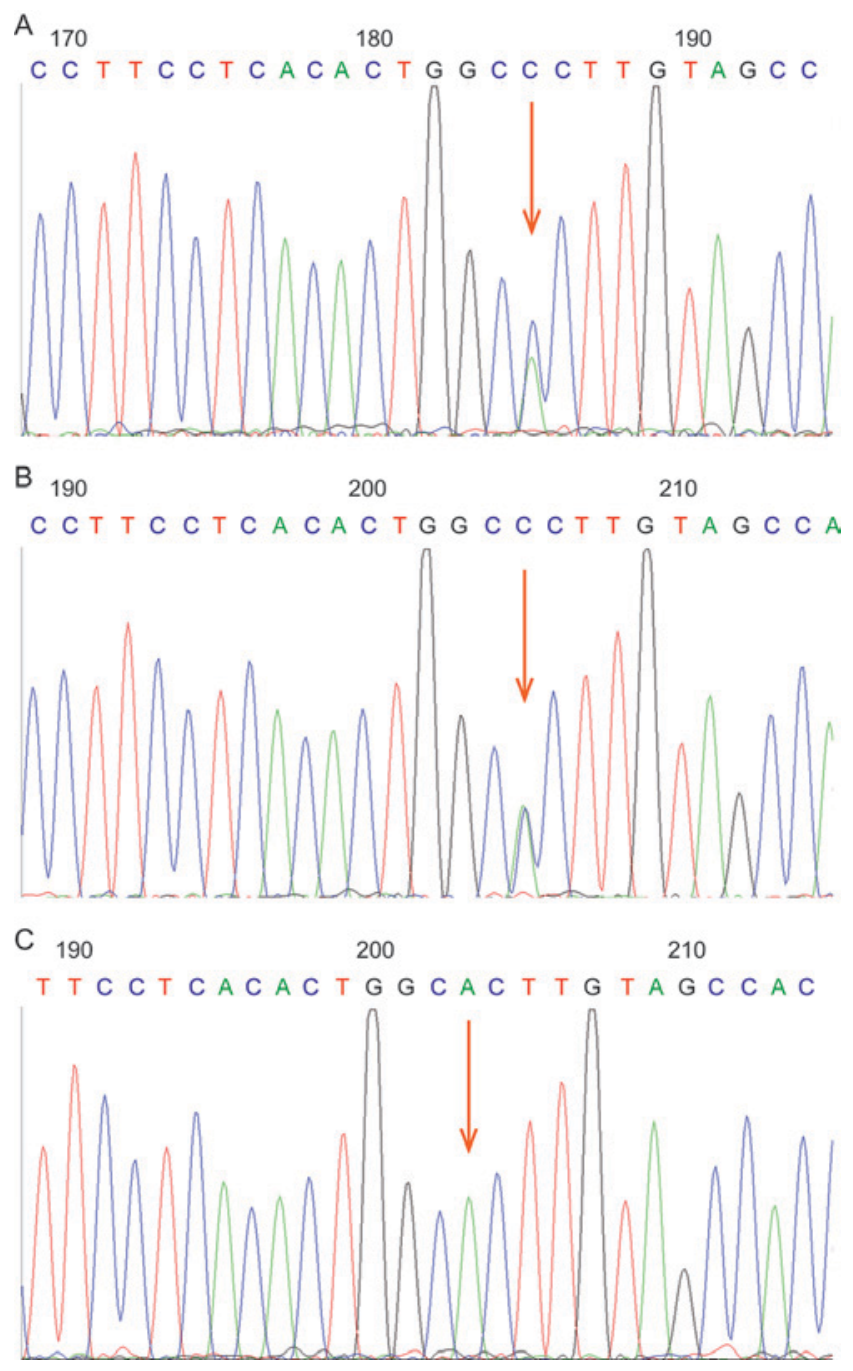

Figure 2. Genetic screening results. Heterozygous missense mutation in exon 8 of the LDLR gene of the proband and her parents. There is a missense mutation in exon 8, c. $28893 \mathrm{~T}>\mathrm{G}$, p. Cys 377 Gly, in both the (A) proband and her (B) mother, whereas the mutation is not present for her (C) father. Arrows indicate the missense mutation.

and the proband were close to 1 at the 1st site, whereas the mother was close to 2 at the 1st site. The results for the 2 nd site demonstrated that the ratios for the parents and the proband were all close to 2 (Fig. 3). These results indicated that the father and proband were haploid at the 1st site, however diploid at the 2 nd site, whereas the mother was diploid at both the 1st and 2 nd sites. This suggests that there
Table III. Mismatched alleles in the low-density lipoprotein-receptor gene exon of the family detected by DNA sequencing.

\begin{tabular}{lcccc}
\hline Exon & Nucleotide (location) & Proband & Father & Mother \\
\hline Exon 16 & rs13306501 & GG & AA & GG \\
Exon 16 & rs2304182 & GG & AA & GG \\
Exon 18 & rs2116899 & GG & AA & GG \\
Exon 18 & rs2116897 & GG & AA & GG \\
Exon 18 & rs2116898 & GG & AA & GG \\
Exon 18 & rs14158 & GG & AA & GG \\
\hline
\end{tabular}
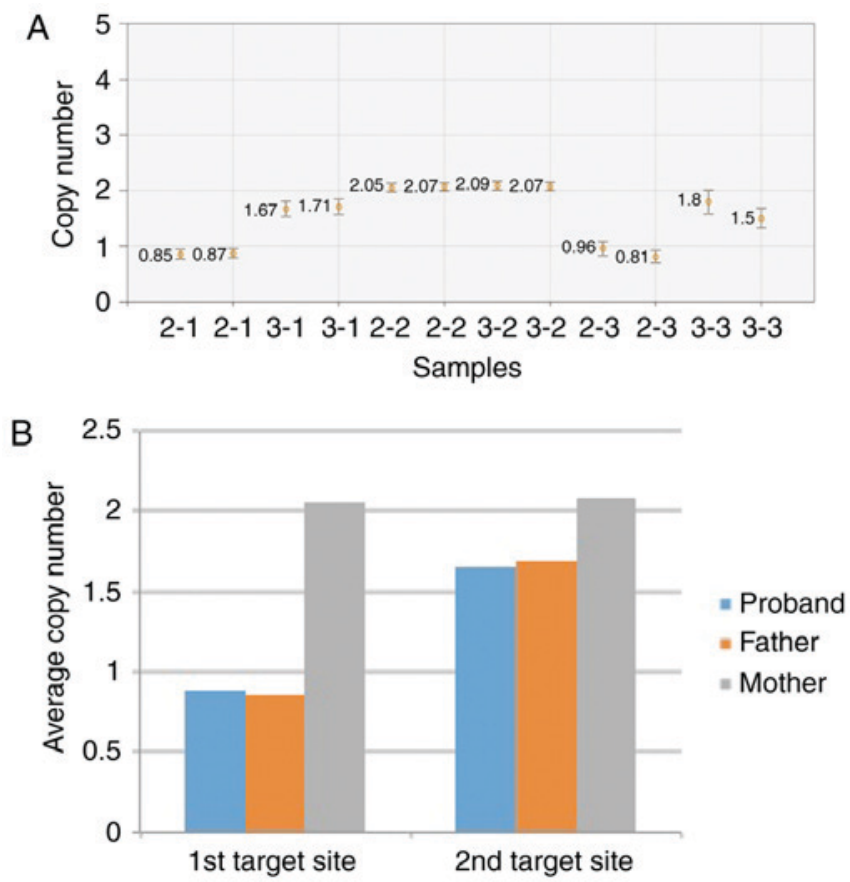

Figure 3. The results from the ddPCR ${ }^{\mathrm{TM}}$ system. (A) The results from the ddPCR $^{\text {TM }}$ system, including the 1st and the 2 nd site of the proband and her parents. 2-1 and 3-1 indicate the 1st and 2nd site of the proband, respectively; whereas, 2-2 and 3-2 indicate her mother's site, and 2-3 and 3-3 represent her father's sites. (B) Quantitative results of part A. QX200 Droplet Digital PCR; ddPCR ${ }^{\mathrm{TM}}$.

were DNA fragment deletions at exon 18 in both the proband and her father. The genotype of this proband belongs to the compound heterozygous FH category. 

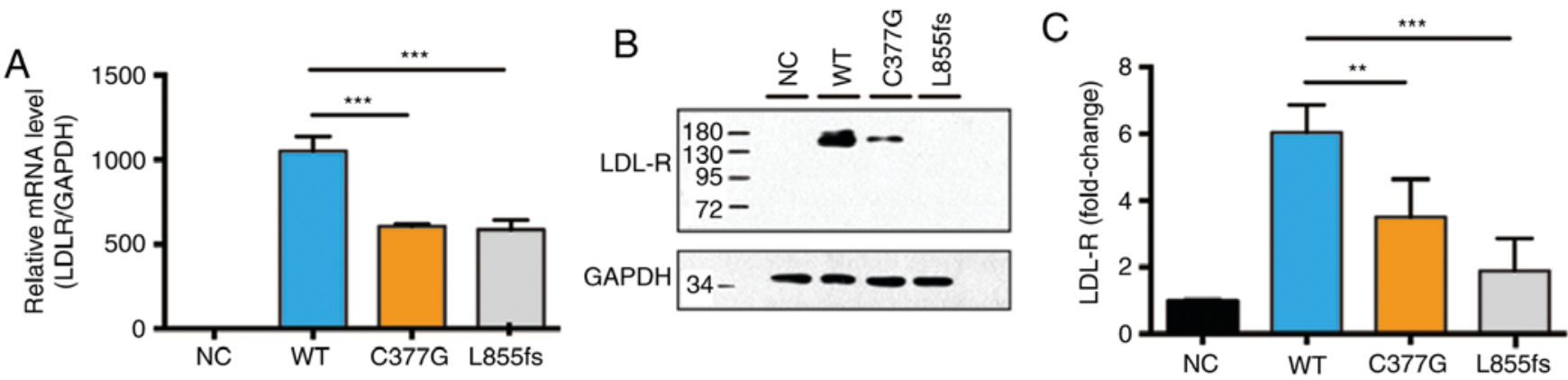

D NC
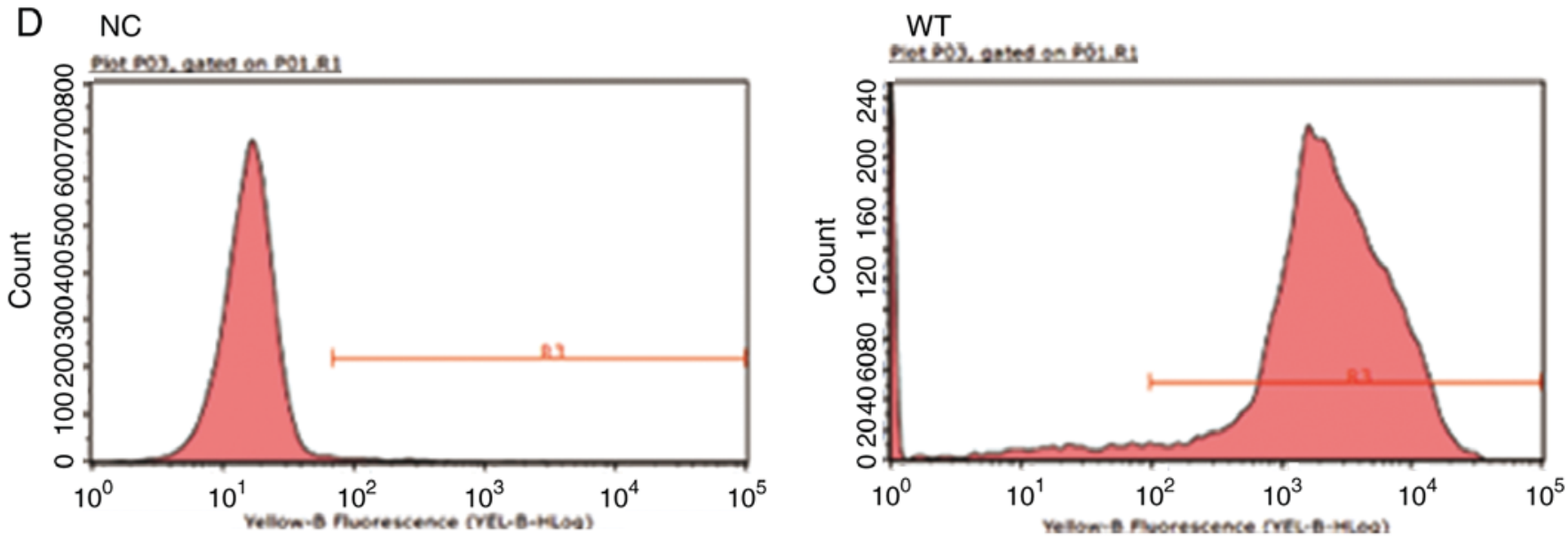

C377G
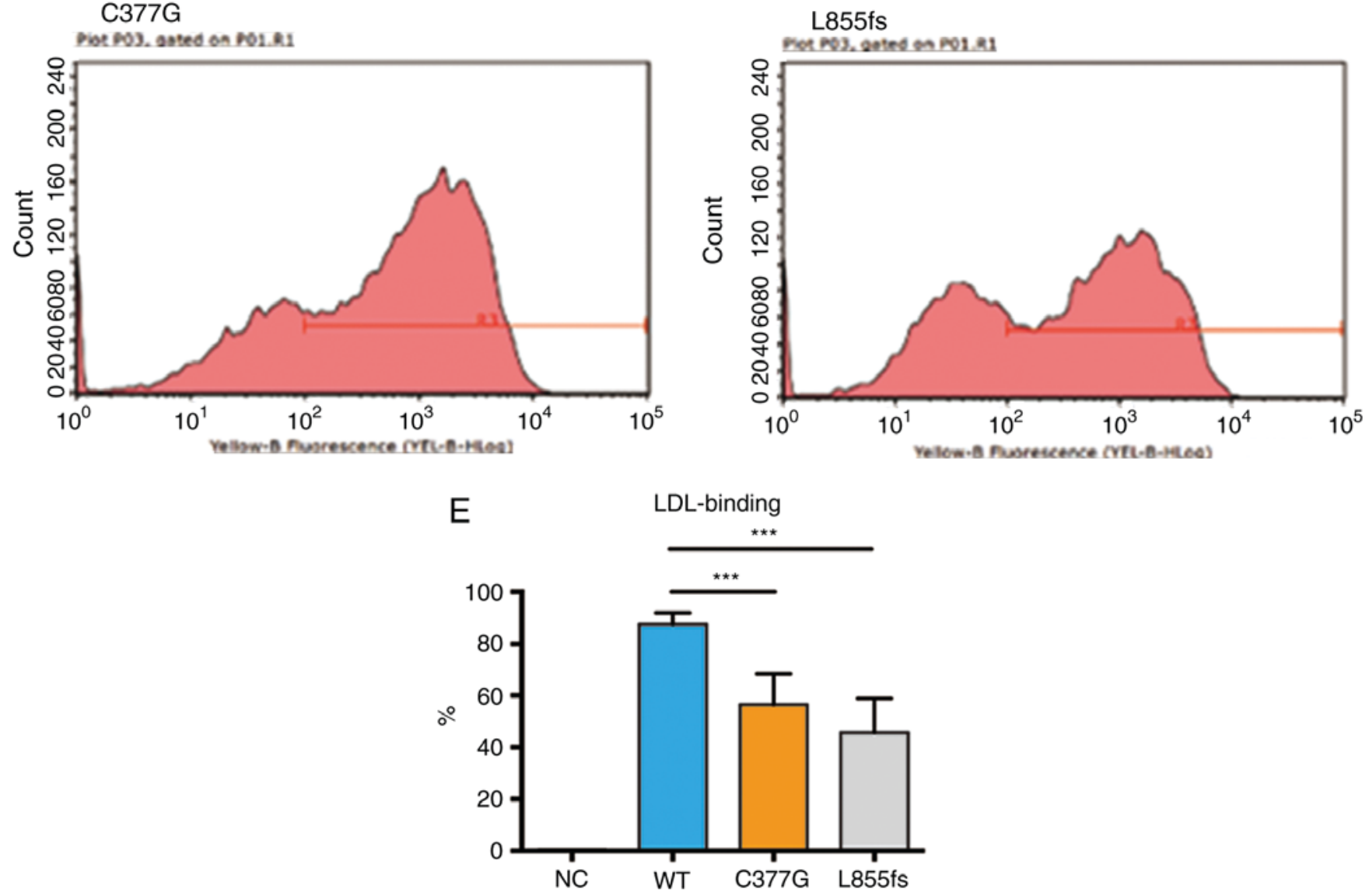

Figure 4. Novel mutations impair LDLR expression and function. (A) Quantitative polymerase chain reaction results of 293T cells transfected with WT, C377G, L855fs mutants and the negative control vector. (B) Western blot analysis of total cell lysates from cells transfected with WT and both C377G and L855fs mutants, as well as the negative control vector. (C) Histogram of different samples in western blotting analysis. (D) Characterization of LDLR binding functions in transfected 293T cells using flow cytometric measurements. Cells were transfected with control vector, WT LDLR, C377G and L855fs mutant respectively. The activity of Dil-LDL binding is given on the x-axis. (E) The histogram exhibits the data as the percentage of mean fluorescence compared with the WT. Bars and vertical lines indicate the mean of five independent measurements and standard deviation, respectively. 
F NC

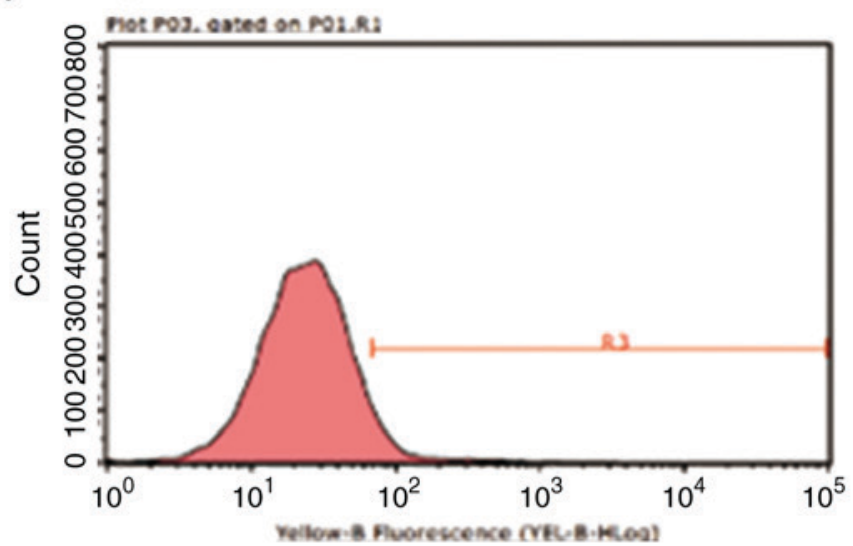

C377G

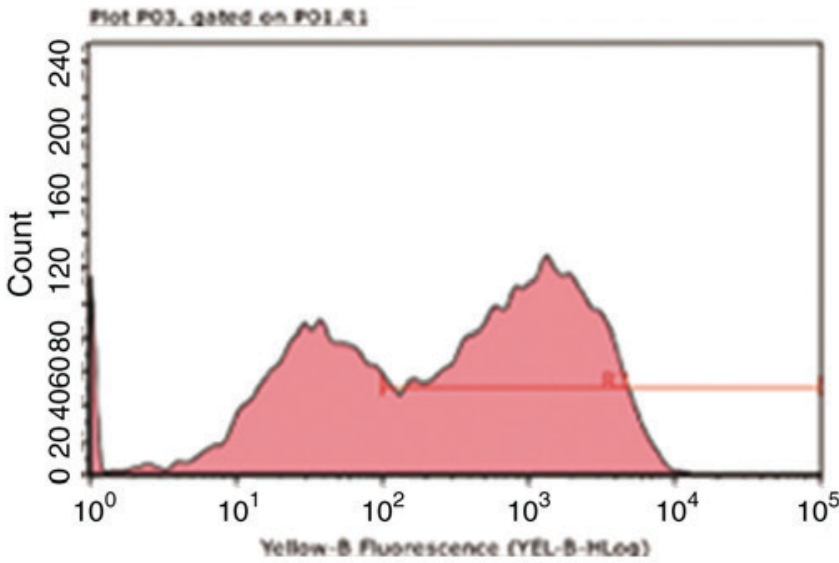

WT

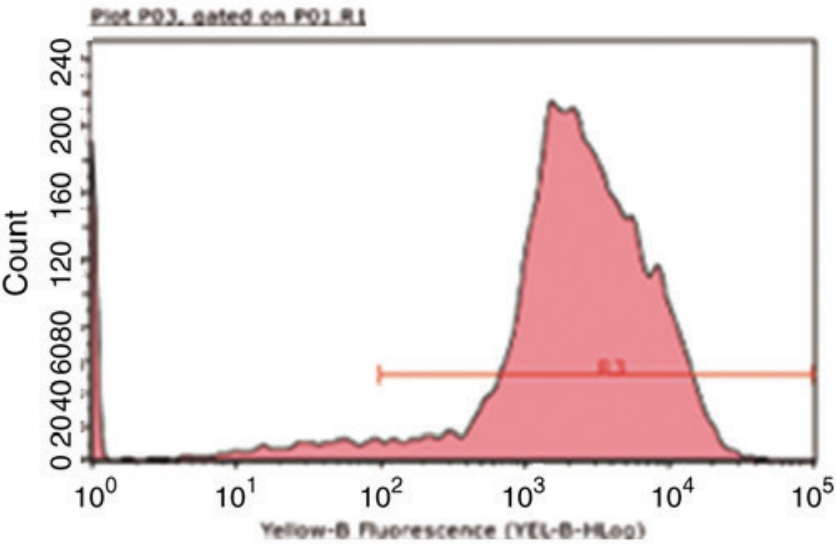

L855fs

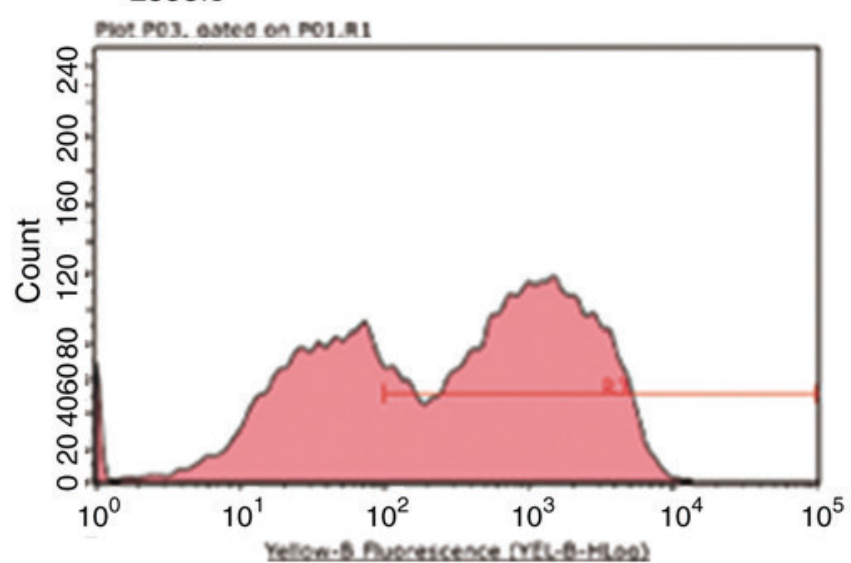

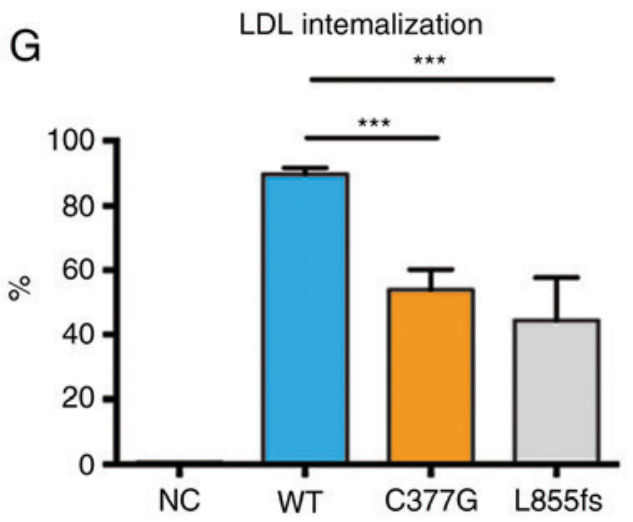

Figure 4. Continued. (F) Characterization of LDLR internalization in transfected 293T cells using flow cytometric measurements. Cells were transfected with control vector, WT LDLR, C377G and L855fs mutant respectively. The activity of Dil-LDL internalizing is given on the x-axis. (G) Histogram of different samples as described previously. ${ }^{* *} \mathrm{P}<0.01 ;{ }^{* * *} \mathrm{P}<0.001$. WT, wild type; NC, normal control; LDLR, low-density lipoprotein receptor.

Novel genetic mutations and fragment deletions impair LDLR function. qPCR and western blot analysis of total cell lysates from 293 T cells transfected with WT and mutant LDLR expression vectors were performed separately, including both $\mathrm{C} 377 \mathrm{G}$ mutations as well as the L855 deletion mutation. Compared with the WT, both C377G missense mutations and L855 fragment deletion mutations led to impaired expression of LDLR at the mRNA (Fig. 4A) and protein levels (Fig. 4B and C). To further demonstrate the functional activity of these two lesions, 293T cells were used without endogenous LDLR for LDL binding and internalization analyses. Flow cytometry analysis demonstrated that compared with WT control, these two mutations had significantly decreased LDL binding ( $\mathrm{P}<0.001$; Fig. 4D and $\mathrm{E})$ and internalization ability $(\mathrm{P}<0.001$; Fig. 4F and $\mathrm{G})$.

Association between the phenotype and genotype in this FH family. The genotype of this proband was the compound heterozygous FH type, whereas her parents exhibited the simple heterozygous genotype. Correspondingly, the proband demonstrated severe clinical manifestations with an LDL-Cof $<14 \mathrm{mmol} / \mathrm{l}$, tendon xanthoma, premature chronic heart diseases and peripheral vascular disease, all which conformed 
Table IV. Association between the phenotype and genotype in the familial hypercholestraemia family.

\begin{tabular}{|c|c|c|c|c|c|c|c|}
\hline \multirow[b]{2}{*}{ Subject } & \multirow[b]{2}{*}{ Age (year) } & \multicolumn{3}{|c|}{ Phenotype } & \multicolumn{2}{|c|}{ Genotype } & \multirow[b]{2}{*}{ Treatment } \\
\hline & & $\mathrm{TC}(\mathrm{mmol} / \mathrm{l})$ & $\begin{array}{l}\text { LDL-C } \\
(\mathrm{mmol} / \mathrm{l})\end{array}$ & Xanthoma & $\mathrm{C} 377 \mathrm{G} 28893 \mathrm{~T}>\mathrm{G}$ & $\begin{array}{l}\text { DNA fragments } \\
\text { deletions at exon } 18\end{array}$ & \\
\hline Proband & 16 & 14.21 & 12.22 & Yes & Yes & Yes & $\begin{array}{l}\text { Atorvastatin } \\
40 \mathrm{mg} / \text { day }\end{array}$ \\
\hline Father & 47 & 7.34 & 4.40 & None & None & Yes & None \\
\hline Mother & 47 & 8.33 & 4.98 & None & Yes & None & None \\
\hline
\end{tabular}

TC, total cholesterol; LDL-C, low-density lipoprotein cholesterol.

to the homozygous FH phenotype. Her parents only demonstrated mildly elevated serum lipid levels with LDL-C between 4.0-4.9 mmol/1, without any other clinical features, which met the heterozygous $\mathrm{FH}$ phenotype, in accordance with The Dutch Lipid Network Criteria (13). Coincidentally, the phenotype was associated with the genotype in this $\mathrm{FH}$ family (Table IV).

\section{Discussion}

The present study covered both phenotypic and genotypic analyses of an FH patient and her family members. The proband in the present study had the typical clinical manifestations of homozygous $\mathrm{FH}$, presenting with xanthoma at the age of three; an LDL-C $>14 \mathrm{mmol} / \mathrm{l}$ at the age of six despite optimal Lipitor treatment; and early onset of severe supravalvular aortic stenosis, carotid artery atherosclerotic plaques and coronary atherosclerosis. Her diagnosis clearly conformed to the homozygous FH clinical diagnostic criteria (14-17).

Based on clinical features, genetic testing is helpful to confirm the diagnosis of homozygous FH. Currently, $>90 \%$ of the diagnosed molecular FH cases result from LDLR genetic mutations (18), whereas the others are primarily due to a pathogenic mutation in apoB or PCSK9 $(19,20)$. All the aforementioned defects will ultimately affect lipid metabolism and result in higher circulating LDL-C concentrations. Van der Graaf et al (21), analyzed 269 pediatric cases of children diagnosed with FH due to gene defects. The results demonstrated that LDLR mutations accounted for $95 \%$ of genetic mutations, whereas apoB mutations accounted for 5\% and no PCSK9 mutations were detected.

Sequencing results, obtained using PCR to amplify the entire sequence of the amino acid coding region of the LDLR gene, including 18 exons in the proband, exhibited a heterozygous mutation in exon 8 (c. 28893 T>G, p. Cys 377 Gly), which resulted in a substitution of Cys at codon 377 with Gly. LDLR gene sequencing of exon 8 of the proband's mother revealed the same genotype, whereas her father had no mutation at that site. Both the proband and her mother's genotypes meet the criteria for heterozygous FH diagnosis. However, the clinical manifestations of the proband are more severe and conform to the homozygous FH diagnosis, whereas those of her mother are consistent with heterozygous FH. Therefore, 18 exons of the LDLR gene of the parents were sequenced for comparison with normal sequences. It was demonstrated that the proband and her parents' partial genes mismatched in exon 16 and exon 18. For the father, multiple alleles presented with AA, whereas GG was present in the mother and proband (rs13306501, rs2304182, rs2116899 and rs2116898). The results from the QX200 ddPCR ${ }^{\mathrm{TM}}$ system demonstrated that the patient and her father were haploid in part of exon 18, indicating DNA fragment deletions at these loci. The results revealed that the proband is compound heterozygous with gene variation. The proband inherited a heterozygous missense mutation in exon 8 of the LDLR gene from her mother, and from her father, she inherited LDLR gene DNA fragment deletions and variation in exon 18. As both of her chromosomes are defective, she cannot make normal LDLR. In fact, the proband's gene variation conforms to the diagnosis of compound heterozygous $\mathrm{FH}$, leading to clinical manifestations in line with the homozygous FH phenotype.

The LDLR gene is located on the short arm of chromosome 19 (Ch19pl3.1-13.3) and has a total length of $45 \mathrm{~kb}$, including 18 exons and 17 introns, which encodes an 839 amino acid precursor protein with seven domains $(22,23)$. According to the Britain FH LDLR gene mutations database (http://www. ucl.ac.uk/ldlr/LOVDv.1.1.0/), >1700 LDLR gene mutations have been reported worldwide. The heterozygous gene mutation of the proband and her mother was previously unknown (c. $28893 \mathrm{~T}>\mathrm{G}, \mathrm{p}$. Cys 377 Gly); namely, this mutation in the LDLR gene sequence coding region (CDS) at the 8th exon, no. 28,893 , leads to a alteration in the encoded protein, as Cys is replaced with Gly. This type of mutation is located in the epidermal growth factor precursor domain and it may lead to transport disorders of LDLR from the endoplasmic reticulum to the Golgi, loss of function for binding to LDL, or LDLR recycling disorders. A previous study revealed that a pathogenic c. $1129 \mathrm{~T}>\mathrm{G}, \mathrm{C} 356 \mathrm{G}$ mutation in exon 8 results in $57 \%$ LDLR binding and $52 \%$ internalization activity in transfected 293T cells (24).

The proband and her father's LDLR gene sequence CDS of exon 18 exhibited deletion mutations, which occurred in the CDS of the transmembrane domain and the cytoplasmic domains. These deletions may easily lead to an inward deficient shift, where LDLR may bind to LDL, however LDL cannot be transported to the cell surface and accumulate in the coating lacuna. This kind of deletion is identical to FH-Helsinki, 
which is a deletion of the LDLR gene that deletes $9.6 \mathrm{~kb}$ from intron 15 to exon 18 and is very common among Finnish FH subjects in Oslo, Norway $(25,26)$. Furthermore, in the present study, analyses using $293 \mathrm{~T}$ cells further demonstrated that both the $\mathrm{C} 377 \mathrm{G}$ mutation and the fragment deletions in exon 18 could affect expression and function of LDL-R to some extent, leading to impaired LDL binding and internalization. However, as the in vitro cell model represents a simplified molecular environment compared with in vivo conditions, such mutation and fragment deletions may cause more significant dysfunction of LDL-R in in vivo experiments.

The proband inherited her mother's exon 8 heterozygous mutation (c. 28893 T>G, p. Cys 377 Gly) and inherited her father's exon 18 deletion mutation, which resulted in two DNA strands that were unable to synthesize normal LDLR. Therefore, she suffered from tendon xanthoma at an early age, a large accumulation of LDL in plasma and severe atherosclerosis during youth.

Regarding pathogenesis, due to a congenital deficiency of LDLR, high amounts of cholesterol accumulated in the body organs, resulting in severe supravalvular aortic stenosis, carotid artery atherosclerotic plaques, coronary atherosclerosis and tendon xanthoma. Given that patients with homozygous FH have a severe or total deficiency in LDLR function, their response to LDL lowering therapies is usually markedly attenuated (27). Although the proband did not reach the target level of LDL during her 13 years of treatment, her LDL level still decreased, through the application of statins. Statins and ezetimibe are able to lower LDL-C in homozygous FH patients by potentially decreasing hepatic secretion of apoB $(1,28)$ and upregulation of hepatic LDLRs (27). However, most of the homozygous FH patients usually require apheresis $(1,29)$. Therefore, it was suggested that the proband take $100 \mathrm{mg}$ aspirin daily, $40 \mathrm{mg}$ Lipitor every night, $10 \mathrm{mg}$ ezetimibe daily and LDL apheresis, based on her LDL level. As the proband currently has no clinical symptoms of dizziness or chest tightness and no coronary ostial lesions, she will be followed-up and surgery only suggested when obvious clinical manifestations appear.

In the present study, the proband's mother had a heterozygous mutation in exon 8 (c. 28893 T>G, p. Cys 377 Gly); this genotype belongs to the heterozygous FH diagnosis and clinically manifests with mildly elevated blood lipids. The patient's father had a single-stranded deletion in exon 18, causing him to belong to the heterozygous FH diagnosis, either with mildly elevated blood lipids or without clinical features upon physical examination.

The proband inherited the variant genes of both her father and her mother, so her genotype and clinical phenotype are consistent with homozygous FH. She exhibited all of the typical symptoms, including early manifestations of xanthoma, hyperlipidemia, cardiovascular disease, ineffective administration of lipid-lowering drugs and a need to reduce the level of cholesterol with apheresis. To some extent, this indicated that clinical manifestations and genotypes are associated. However, HF is a heterogeneous disease with different mutations and the same site mutation has occurred in different patients, however with different clinical manifestations and symptoms of different severity, particularly in those of the heterozygous genotype. Epidemiological studies demonstrated that the clinical manifestations of $\mathrm{FH}$ heterozygotes in China are much less severe compared with patients in the West, which may be associated with the traditional low-fat diet and some of the 'lipid-regulating' genes in China $(1,28)$.

Regardless, once identified, FH patients should be prescribed a high-potency statin therapy at the highest tolerated dose. For the severe homozygous FH patients, lipoprotein apheresis is further administered to remove lipoproteins. Several studies have demonstrated that statins and lipoprotein apheresis may reduce the progression of atherosclerosis and improve the outcome of FH (30-33). Therefore, early diagnosis and early treatment may delay the cumulative burden of elevated LDL-C, reducing the risk of complications associated with atherosclerosis and coronary heart disease (34). Therefore, clinicians and society should raise awareness of $\mathrm{FH}$ and the comprehensive screening strategy, including the clinical and genetic aspects, in order to significantly improve the outcomes for these patients $(17,35)$.

In conclusion, in the present study, a compound heterozygous family with FH were investigated. The family members carried genetic defects in exon 8 , with a heterozygous mutation (c. 28893 T>G, p. Cys 377 Gly) in one chromosome strand, and a deletion mutation in exon 18 in another chromosome strand. To the best of the author's knowledge, the heterozygous allelic mutation has not yet been reported. The proband's genotype is different from any of the previously reported $\mathrm{FH}$ genetic variations. The genotype is associated with the phenotype, which may help to reasonably explain the clinical manifestations.

\section{Acknowledgements}

The authors wish to acknowledge the contribution of all the doctors of the Department of Cardiology at Rui Jin Hospital, Shanghai Jiao Tong University School of Medicine, (Shanghai, China) to the present study.

\section{Funding}

The present study was supported by the National Natural Science Foundation of China (grant no. 81670352 to RT), Shanghai Municipal Education Commission-Gaofeng Clinical Medicine Grant Support (grant no. 20152205 to RT) and Shanghai Municipal Commission of Health and Family Planning (grant no. 201740248 to FW). No relationships with industry are to be declared.

\section{Availability of data and materials}

The analyzed data sets generated during the study are available from the corresponding author on reasonable request.

\section{Authors' contributions}

FW, QF and RX contributed to all stages of the present study, including conception and design of the study, as well as the acquisition, analysis and interpretation of data. RT, GG and RZ performed the experiments and assisted with data acquisition, analysis and interpretation of the data. FW and QF contributed to the writing of the article, together with RT, RX, RZ and GG 
who additionally revised the manuscript. All authors read and approved the final manuscript.

\section{Ethics approval and consent to participate}

All investigated subjects gave written informed consent for all these tests and the publication of associated data and accompanying images prior to taking part in the study and participating in these examinations. The study was conducted according to the Helsinki declaration (2013) and approved by the Institutional Ethics Committees of Shanghai Jiao Tong University, affiliated with Rui Jin Hospital (Shanghai, China).

\section{Consent for publication}

All investigated subjects gave written informed consent for publication.

\section{Competing interests}

The authors declare that they have no competing interests.

\section{References}

1. Raal FJ and Santos RD: Homozygous familial hypercholesterolemia: Current perspectives on diagnosis and treatment. Atherosclerosis 223: 262-268, 2012.

2. Goldstein JL, Hobbs HH and Brown MS: Familial hypercholesterolemia. In: The metabolic basis of inherited disease. McGraw-Hill, New York, pp2863-2913, 2001.

3. Wang L, Lin J, Liu S, Cao S, Liu J, Yong Q, Yang Y, Wu B, Pan X, Du L, et al: Mutations in the LDL receptor gene in four Chinese homozygous familial hypercholesterolemia phenotype patients. Nutr Metab Cardiovasc Dis 19: 391-400, 2009.

4. Hooper AJ, Nguyen LT, Burnett JR, Bates TR, Bell DA, Redgrave TG, Watts GF and van Bockxmeer FM: Genetic analysis of familial hypercholesterolaemia in Western Australia. Atherosclerosis 224: 430-434, 2012.

5. Jiang L, Sun LY, Dai YF, Yang SW, Zhang F and Wang LY: The distribution and characteristics of LDL receptor mutations in China: A systematic review. Sci Rep 5: 17272, 2015.

6. Humphries SE, Norbury G, Leigh S, Hadfield SG and Nair D: What is the clinical utility of DNA testing in patients with familial hypercholesterolaemia? Curr Opin Lipidol 19: 362-368, 2008.

7. Taylor A, Wang D, Patel K, Whittall R, Wood G, Farrer M, Neely RD, Fairgrieve S, Nair D, Barbir M, et al: Mutation detection rate and spectrum in familial hypercholesterolaemia patients in the UK pilot cascade project. Clin Genet 77: 572-580, 2010.

8. Lombardi MP, Redeker EJ, van Gent DH, Smeele KL, Weerdesteijn R and Mannens MM: Molecular genetic testing for familial hypercholesterolemia in the Netherlands: A stepwise screening strategy enhances the mutation detection rate. Genet Test 10: 77-84, 2006.

9. Poncz M, Solowiejczyk D, Harpel B, Mory Y, Schwartz E and Surrey S: Construction of human gene libraries from small amounts of peripheral blood: Analysis of beta-like globin genes. Hemoglobin 6: 27-36, 1982.

10. Belgrader P, Tanner SC, Regan JF, Koehler R, Hindson BJ and Brown AS: Droplet digital PCR measurement of HER2 copy number alteration in formalin-fixed paraffin-embedded breast carcinoma tissue. Clin Chem 59: 991-994, 2013.

11. Hindson BJ, Ness KD, Masquelier DA, Belgrader P, Heredia NJ, Makarewicz AJ, Bright IJ, Lucero MY, Hiddessen AL, Legler TC, et al: High-throughput droplet digital PCR system for absolute quantitation of DNA copy number. Anal Chem 83 . 8604-8610, 2011

12. Livak KJ and Schmittgen TD: Analysis of relative gene expression data using real-time quantitative PCR and the 2(-Delta Delta C(T)) method. Methods 25: 402-408, 2001.
13. Hartgers ML, Ray KK and Hovingh GK: New approaches in detection and treatment of familial hypercholesterolemia. Curr Cardiol Rep 17: 109, 2015.

14. Watts GF, Sullivan DR, Poplawski N, van Bockxmeer F, Hamilton-Craig I, Clifton PM, O'Brien R, Bishop W, George P, Barter PJ, et al: Familial hypercholesterolaemia: A model of care for Australasia. Atheroscler Suppl 12: 221-263, 2011.

15. Harada-Shiba M, Arai H, Oikawa S, Ohta T, Okada T, Okamura T, Nohara A, Bujo H, Yokote K, Wakatsuki A, et al: Guidelines for the management of familial hypercholesterolemia. J Atheroscler Thromb 19: 1043-1060, 2012

16. Williams RR, Hunt SC, Schumacher MC, Hegele RA, Leppert MF, Ludwig EH and Hopkins PN: Diagnosing heterozygous familial hypercholesterolemia using new practical criteria validated by molecular genetics. Am J Cardiol 72: 171-176, 1993.

17. Watts GF, Gidding S, Wierzbicki AS, Toth PP, Alonso R, Brown WV, Bruckert E, Defesche J, Lin KK, Livingston M, et al: Integrated guidance on the care of familial hypercholesterolaemia from the International FH Foundation: Executive summary. J Atheroscler Thromb 21: 368-374, 2014.

18. Nordestgaard BG, Chapman MJ, Humphries SE, Ginsberg HN, Masana L, Descamps OS, Wiklund O, Hegele RA, Raal FJ, Defesche JC, et al: Familial hypercholesterolaemia is underdiagnosed and undertreated in the general population: Guidance for clinicians to prevent coronary heart disease: Consensus statement of the European Atherosclerosis Society. Eur Heart J 34: a3478-a3490, 2013.

19. Tybjaerg-Hansen A and Humphries SE: Familial defective apolipoprotein B-100: A single mutation that causes hypercholesterolemia and premature coronary artery disease. Atherosclerosis 96: 91-107, 1992

20. Abifadel M, Varret M, Rabès JP, Allard D, Ouguerram K, Devillers M, Cruaud C, Benjannet S, Wickham L, Erlich D, et al: Mutations in PCSK9 cause autosomal dominant hypercholesterolemia. Nat Genet 34: 154-156, 2003.

21. van der Graaf A, Avis HJ, Kusters DM, Vissers MN, Hutten BA, Defesche JC, Huijgen R, Fouchier SW, Wijburg FA, Kastelein JJ and Wiegman A: Molecular basis of autosomal dominant hypercholesterolemia: Assessment in a large cohort of hypercholesterolemic children. Circulation 123: 1167-1173, 2011.

22. Lindgren V, Luskey KL, Russell DW and Francke U: Human genes involved in cholesterol metabolism: Chromosomal mapping of the loci for the low density lipoprotein receptor and 3-hydroxy-3-methylglutaryl-coenzyme A reductase with cDNA probes. Proc Natl Acad Sci USA 82: 8567-8571, 1985.

23. Yamamoto T, Davis CG, Brown MS, Schneider WJ, Casey ML, Goldstein JL and Russell DW: The human LDL receptor: A cysteine-rich protein with multiple Alu sequences in its mRNA. Cell 39: 27-38, 1984.

24. Wu WF, Sun LY, Pan XD, Yang SW and Wang LY: Use of targeted exome sequencing in genetic diagnosis of Chinese familial hypercholesterolemia. PLoS One 9: e94697, 2014.

25. Rødningen OK, Tonstad S, Ose L, Berg K and Leren TP: Effects of a 9.6-kb deletion of the LDL receptor gene (FH Helsinki) on structure and levels of mRNA. Hum Mutat 12: 95-102, 1998.

26. Rødningen OK, Røsby O, Tonstad S, Ose L, Berg K and Leren TP: A 9.6 kilobase deletion in the low density lipoprotein receptor gene in Norwegian familial hypercholesterolemia subjects. Clin Genet 42: 288-295, 1992.

27. Ooi EM, Barrett PH and Watts GF: The extended abnormalities in lipoprotein metabolism in familial hypercholesterolemia: Developing a new framework for future therapies. Int J Cardiol 168: 1811-1818, 2013

28. Gagné C, Gaudet D and Bruckert E; Ezetimibe Study G: Efficacy and safety of ezetimibe coadministered with atorvastatin or simvastatin in patients with homozygous familial hypercholesterolemia. Circulation 105: 2469-2475, 2002.

29. Thompson GR, Catapano A, Saheb S, Atassi-Dumont M, Barbir M, Eriksson M, Paulweber B, Sijbrands E, Stalenhoef AF and Parhofer KG: Severe hypercholesterolaemia: Therapeutic goals and eligibility criteria for LDL apheresis in Europe. Curr Opin Lipidol 21: 492-498, 2010.

30. Smilde TJ, van Wissen S, Wollersheim H, Trip MD, Kastelein JJ and Stalenhoef AF: Effect of aggressive versus conventional lipid lowering on atherosclerosis progression in familial hypercholesterolaemia (ASAP): A prospective, randomised, double-blind trial. Lancet 357: 577-581, 2001. 
31. Versmissen J, Oosterveer DM, Yazdanpanah M, Defesche JC, Basart DC, Liem AH, Heeringa J, Witteman JC, Lansberg PJ, Kastelein JJ and Sijbrands EJ: Efficacy of statins in familial hypercholesterolaemia: A long term cohort study. BMJ 337: a2423, 2008.

32. Raal FJ, Pilcher GJ, Panz VR, van Deventer HE, Brice BC, Blom DJ and Marais AD: Reduction in mortality in subjects with homozygous familial hypercholesterolemia associated with advances in lipid-lowering therapy. Circulation 124: 2202-2207, 2011.

33. Thompson GR: The evidence-base for the efficacy of lipoprotein apheresis in combating cardiovascular disease. Atheroscler Suppl 14: 67-70, 2013.
34. Wiegman A, Gidding SS, Watts GF, Chapman MJ, Ginsberg HN, Cuchel M, Ose L, Averna M, Boileau C, Borén J, et al: Familial hypercholesterolaemia in children and adolescents: Gaining decades of life by optimizing detection and treatment. Eur Heart J 36: 2425-2437, 2015 .

35. Cuchel M, Bruckert E, Ginsberg HN, Raal FJ, Santos RD, Hegele RA, Kuivenhoven JA, Nordestgaard BG, Descamps OS, Steinhagen-Thiessen E, et al: Homozygous familial hypercholesterolaemia: New insights and guidance for clinicians to improve detection and clinical management. A position paper from the Consensus Panel on Familial Hypercholesterolaemia of the European Atherosclerosis Society. Eur Heart J 35: 2146-2157, 2014. 